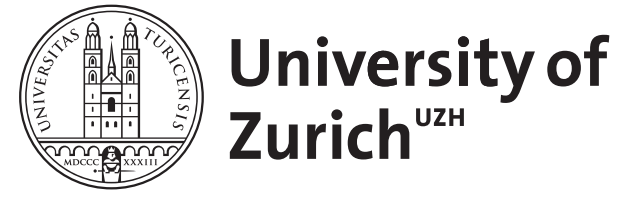
Archive

University of Zurich

University Library

Strickhofstrasse 39

CH-8057 Zurich

www.zora.uzh.ch

Year: 1987

\title{
Stable bundles on regular elliptic surfaces
}

Lübke, M ; Okonek, C

DOI: https://doi.org/10.1515/crll.1987.378.32

Posted at the Zurich Open Repository and Archive, University of Zurich

ZORA URL: https://doi.org/10.5167/uzh-22992

Journal Article

Published Version

Originally published at:

Lübke, M; Okonek, C (1987). Stable bundles on regular elliptic surfaces. Journal für die Reine und Angewandte Mathematik, 378:32-45.

DOI: https://doi.org/10.1515/crll.1987.378.32 


\title{
Stable bundles on regular elliptic surfaces
}

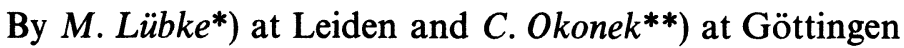

\section{Introduction}

In this paper we study stable and simple vector bundles on simply connected elliptic surfaces.

Let $\pi: X \rightarrow \mathbb{P}_{1}$ be such a surface with geometric genus $p_{g}$. If $p_{g}=0$, then $X$ is a projective algebraic surface. Moduli spaces of stable rank-2 bundles on these surfaces have been computed in [21]. Here we extend the techniques from [21] and consider elliptic surfaces of higher geometric genus. Now the surfaces are in general nonalgebraic, so we have to be careful with what we mean by stability.

Since every simply connected elliptic surface has a Kähler structure [17], it makes sense to consider $\omega$-stable bundles for a suitable Kähler form $\omega$ [9]. If $\omega$ is induced by an ample line bundle $L$, this notion coincides with $L$-stability in the sense of MumfordTakemoto.

Furthermore, from the proof of the conjecture of Kobayashi and Hitchin by Uhlenbeck and Yau [23] (see also [4]) we get a one to one correspondence between isomorphism classes of $\omega$-stable bundles and gauge equivalence classes of irreducible projectively anti-self-dual unitary connections (i.e. Yang-Mills connections, i.e. Hermitian-Einstein bundles), so we can use results from Yang-Mills theory.

We adress the following questions:

1) What are the possible second Chern classes of $\omega$-stable and simple rank-2 bundles with trivial determinant on a given surface $X$ ?

2) Can one describe the corresponding moduli spaces?

The expected dimension of the moduli space of stable 2-bundles with Chern classes $c_{1}=0, c_{2}$ is $4 c_{2}-3\left(p_{g}+1\right)$, so one expects such bundles for all $c_{2} \geqq\left[\frac{3}{4}\left(p_{g}+1\right)\right]$. In [22] Taubes proved a general existence theorem for anti-self-dual $S U(2)$-connections over arbitrary Riemannian 4-manifolds, which in our case for generic $\omega$ implies

*) Partially supported by the SFB 170 in Göttingen.

**) Supported by the Heisenberg-program of the DFG. 
existence of $\omega$-stable bundles for $c_{2} \geqq 2 p_{g}+1$. We show, that for a good choice of the Kähler form there exist $\omega$-stable 2-bundles with $c_{1}=0, c_{2}=1$ on all simply connected elliptic surfaces which have at least 2 multiple fibres with multiplicities greater than 1 , thus showing that Taubes bound in [22] is in general not best possible. In fact, this was one of our motivations for this work.

If the surface is algebraic and $\omega$ is induced by a polarization $L$, we compute the moduli spaces of $\omega$-stable 2-bundles with $c_{1}=0, c_{2}=1$. These spaces turn out to be finite disjoint unions of in general nowhere reduced curves. If the surface is nonalgebraic, we get a complex structure in the moduli space of $\omega$-stable bundles in the following way: The moduli space of simple bundles is a (not necessarily Hausdorff) complex space [19], and the $\omega$-stable bundles form a (globally Hausdorff) open subset [14]. We determine the space of simple bundles and see that the (in general proper) subset of $\omega$-stable ones admits the same description as in the algebraic case.

This work was supported by the Sonderforschungsbereich 170 in Göttingen; the authors thank it for the hospitality.

The second author takes this opportunity to thank A. van de Ven who introduced him to the subject.

\section{Rank-2 vector bundles on elliptic Kähler surfaces}

The bundles we shall study will appear as certain extensions, so let us first recall the following general fact from [21]:

Proposition 1. 1. Let $X$ be a compact complex surface with canonical bundle $K, L$ any line bundle on $X$ and $Z \subset X$ a finite set of simple points. Then there exists an extension

$$
0 \rightarrow L \otimes K^{-1} \rightarrow E \rightarrow J_{Z} \otimes L^{-1} \otimes K \rightarrow 0
$$

with $E$ locally free of rank 2, if (and only if in case $Z=\{z\}$ is a single point) $Z$ is contained in the zero-set of every section of $\left(K^{\otimes 3}\right) \otimes\left(L^{-1}\right)^{\otimes 2}$. For given $Z=\{z\}$ and $L$, the bundle $E$ is uniquely determined by the existence of such an extension as soon as $h^{1}\left(L^{\otimes 2} \otimes\left(K^{-1}\right)^{\otimes 2}\right)=0$.

To characterize simple bundles we use

Proposition 1. 2. Let $X, Z$ be as above, $L$ a line bundle on $X$ with $h^{0}\left(J_{Z} \otimes\left(L^{-1}\right)^{\otimes 2} \otimes K^{\otimes 2}\right)=0$, and let $E$ be a bundle defined by an extension (*). Then $E$ is simple if and only if $h^{0}\left(L^{\otimes 2} \otimes\left(K^{-1}\right)^{\otimes 2}\right)=0$.

Proof. From $(*)$ one sees $c_{1}(E)=0$ and therefore $E^{*}=E$. Thus by tensoring with $E$ we get the exact sequence

$$
0 \rightarrow E \otimes L \otimes K^{-1} \rightarrow \text { End } E \rightarrow J_{Z} \otimes E \otimes L^{-1} \otimes K \rightarrow 0
$$

with $H^{0}($ End $E) \rightarrow H^{0}\left(J_{Z} \otimes E \otimes L^{-1} \otimes K\right)$ non-zero. 
Since the last vector space is 1-dimensional, $E$ is simple if and only if

$$
h^{0}\left(L^{\otimes 2} \otimes\left(K^{-1}\right)^{\otimes 2}\right)=h^{0}\left(E \otimes L \otimes K^{-1}\right)=0 .
$$

Now let $X$ be a compact Kähler surface with Kähler form $\omega$. As in [9], [13] we define $\omega$-(semi-)stability as follows: Let $F$ be a coherent torsion-free sheaf of rank $r$ on $X$; then $\operatorname{det} F=\left(\Lambda^{r} F\right)^{* *}$ is a line bundle. Choose a hermitian metric $h$ in $\operatorname{det} F$ and consider the first Chern form $c_{1}(\operatorname{det} F, h)$ associated to the metric connection. Now define the $\omega$-degree of $F$ by

$$
\operatorname{deg}_{\omega}(F)=\int_{X} c_{1}(\operatorname{det} F, h) \wedge \omega .
$$

Note that this number depends on the Kähler class of $\omega$, but not on the metric in $\operatorname{det} F$; we often omit the subscript $\omega$ if it is clear which Kähler class we use.

Definition. $F$ is called $\omega$-(semi-)stable if for every rank-s subsheaf $F^{\prime} \subset F$ with $0<s<r$ one has

$$
\frac{\operatorname{deg}\left(F^{\prime}\right)}{s}<\frac{\operatorname{deg}(F)}{r} \quad\left(\frac{\operatorname{deg}\left(F^{\prime}\right)}{s} \leqq \frac{\operatorname{deg}(F)}{r}\right) .
$$

If $\omega$ is induced by an ample line bundle $L$, then $\omega$-stability is the same as $L$-stability. Of course every $\omega$-stable bundle is simple. We use the following criterion for $\omega$-(semi-) stability:

Proposition 1. 3. Let $L, Z$ be as before with $\operatorname{deg}\left(L \otimes K^{-1}\right)<0 \quad(\leqq 0)$,

$$
h^{0}\left(J_{Z} \otimes\left(L^{-1}\right)^{\otimes 2} \otimes K^{\otimes 2}\right)=0,
$$

and let $E$ be a bundle defined by extension (*). Then $E$ is $\omega$-(semi-)stable if and only if for every curve $R$ containing $Z$ with $h^{0}\left(E \otimes L \otimes K^{-1} \otimes \mathcal{O}(R)\right) \neq 0$ one has

$$
\operatorname{deg}\left(L \otimes K^{-1} \otimes \mathcal{O}(R)\right)>0 \quad(\geqq 0) .
$$

Proof. From (*) we get $\operatorname{deg}(E)=0$, so $E$ is not stable if and only if there exists a monomorphism $L_{1} \subseteq E$, where $L_{1}$ is a line bundle with $\operatorname{deg}\left(L_{1}\right) \geqq 0$. Since $\operatorname{deg}\left(L \otimes K^{-1}\right)<0$, this can only happen if there is a curve $R$ containing $Z$ with $\mathcal{O}(R)=L^{-1} \otimes K \otimes L_{1}^{-1}$, hence

$$
\operatorname{deg}\left(L \otimes K^{-1} \otimes \mathcal{O}(R)\right)=\operatorname{deg}\left(L_{1}^{-1}\right) \leqq 0 .
$$

The converse is obvious, as is the semistable case. 
From now on, let $X \rightarrow \mathbb{P}_{1}$ be an elliptic surface, and denote by $F_{p_{i}}, i=1, \ldots, m$, the multiple fibres of $X$ with multiplicities $2 \leqq p=p_{1} \leqq \cdots \leqq p_{m}=q$.

We assume that all fibres are irreducible, $m \geqq 2$, that $X$ has vanishing irregularity $q(X)=0$, and that $X$ admits a Kähler form $\omega_{0}$ (the surfaces in question later all have these properties).

Now the canonical divisor of $X$ is given by

$$
K \sim\left(p_{g}-1\right) F+\sum_{i=1}^{m}\left(p_{i}-1\right) F_{p_{i}}
$$

where $p_{g}=p_{g}(X)$ and $F$ is a generic fibre; in particular we have $K^{2}=0$ [2].

To get a good hold on stable bundles we modify $\omega_{0}$ in the following way: If $\omega_{1}$ is the Kähler form of the Fubini-Study-metric on $\mathbb{P}_{1}$, define

$$
\omega=\omega_{0}+n \pi^{*}\left(\omega_{1}\right)
$$

with a fixed number $n>\max \left(\operatorname{deg}_{\omega_{0}}(K), 1\right)$, where $\pi: X \rightarrow \mathbb{P}_{1}$ is the projection. Then $\omega$ again is a Kähler form, and in this setting we get

Corollary 1.4. If the line bundle $L$ in Proposition 1.3 satisfies $\operatorname{deg}_{\omega_{0}}(L) \geqq 0$ and $\int_{X} c_{1}(L) \wedge \pi^{*}\left(\omega_{1}\right)=0$ (in particular if $L$ is the line bundle associated to a curve $C$ which is vertical, i.e. $C \cdot F=0)$, then a curve $R$ violating the $\omega$-(semi-)stability of $E$ is vertical.

If

$$
L=\mathcal{O}(C), \quad C=a F+\sum_{i=1}^{m} x_{i} F_{p_{i}} \text { and } R=b F+\sum_{i=1}^{m} y_{i} F_{p_{i}}
$$

with $0 \leqq a, b$ and $0 \leqq x_{i}, y_{i}<p_{i}$, then the condition $\operatorname{deg}_{\omega}\left(L \otimes K^{-1} \otimes \mathcal{O}(R)\right)>0(\geqq 0)$ reads

$$
a+b+\sum_{i=1}^{m} \frac{x_{i}+y_{i}+1}{m}-p_{g}-m+1>0 \quad(\geqq 0) .
$$

Proof. First note, that $\pi^{*}\left(\omega_{1}\right)$ is a first Chern form of the line bundle $\mathcal{O}(F)$, so for every vertical curve $C$

$$
\int_{X} c_{1}(\mathcal{O}(C)) \wedge \pi^{*}\left(\omega_{1}\right)=C \cdot F=0
$$

Therefore a necessary condition for $R$ to violate stability is

$$
\begin{aligned}
0 & \geqq \operatorname{deg}_{\omega}\left(L \otimes K^{-1} \otimes \mathcal{O}(R)\right) \\
& =\operatorname{deg}_{\omega_{0}}\left(L \otimes K^{-1} \otimes \mathcal{O}(R)\right)+n \int_{X} c_{1}(R) \wedge \pi^{*}\left(\omega_{1}\right) \\
& \geqq-\operatorname{deg}_{\omega_{0}}(K)+n R \cdot F .
\end{aligned}
$$

Now $R$ is a curve, so $0 \leqq R \cdot F \in \mathbb{Z}$, and the choice of $n$ gives $R \cdot F=0$. 
We are now in the position to prove existence theorems.

Theorem 1.5. If $3 \leqq p(3 \leqq p<q)$, then for all $c_{2} \geqq 1$ there is a simple 2-bundle on $X$ with Chern classes $c_{1}=0, c_{2}$, which is not $\omega$-(semi-)stable.

Remark. For $p=2$ we later shall see examples of surfaces where every simple bundle is stable. For the case $c_{2}=0$ see the remark at the end of this section.

Proof. Let $Z \subset F_{q}$ be a set of simple points of length $c_{2}$. From Proposition 1.1 we get an extension

$$
0 \rightarrow \mathcal{O}\left(F_{p}-F_{q}\right) \rightarrow E \rightarrow J_{Z} \otimes \mathcal{O}\left(F_{q}-F_{p}\right) \rightarrow 0
$$

defining a 2-bundle $E$ with Chern classes $c_{1}=0, c_{2}$, which is not $\omega$-(semi-)stable because

$$
\operatorname{deg}_{\omega}\left(\mathcal{O}\left(F_{p}-F_{q}\right)\right)=\left(\frac{1}{p}-\frac{1}{q}\right) \operatorname{deg}_{\omega_{0}}(F) \geqq 0 \quad(>0) .
$$

That $E$ is simple follows from

$$
h^{0}\left(\mathcal{O}\left(2 F_{p}-2 F_{q}\right)\right)=h^{0}\left(\mathcal{O}\left(2 F_{q}-2 F_{p}\right)\right)=0
$$

and Proposition 1.2.

Theorem 1. 6. If $p<q$, then for all $c_{2} \geqq 1$ there exists an $\omega$-stable 2-bundle $E$ on $X$ with Chern classes $c_{1}=0, c_{2}$; if $p \leqq q$, then for all $c_{2} \geqq 2$ such a bundle exists.

Remark. In the case $p=q=m=2$ (e.g. Enriques surface) there is in fact no stable 2-bundle with $c_{1}=0, c_{2}=1$.

Proof. Let $Z \subset F_{p}$ be a set of simple points of length $c_{2}$, and consider the extension

$$
0 \rightarrow \mathcal{O}\left(-F_{q}\right) \rightarrow E \rightarrow J_{Z} \otimes \mathcal{O}\left(F_{q}\right) \rightarrow 0
$$

which by Proposition 1.1 defines a 2-bundle $E$ with Chern classes $c_{1}=0, c_{2}$. If $E$ were not stable, from Proposition 1.3 and Corollary 1.4 we would find a vertical curve $R$ containing $Z$ with

$$
\operatorname{deg}_{\omega}(\mathcal{O}(R)) \leqq \operatorname{deg}_{\omega}\left(\mathcal{O}\left(F_{q}\right)\right)=\frac{1}{q} \operatorname{deg}_{\dot{\omega}_{0}}(\mathcal{O}(F))
$$
On the other hand, since all fibres are irreducible we can write $R \sim a F+\sum_{i=1}^{m} x_{i} F_{p_{i}}$ with
$0 \leqq a, 0 \leqq x_{i}<p_{i}$, to get

$$
\operatorname{deg}_{\omega}(\mathcal{O}(R))=\left(a+\sum_{i=1}^{m} \frac{x_{i}}{p_{i}}\right) \operatorname{deg}_{\omega_{0}}(\mathcal{O}(F)) .
$$


This implies $a=0$, and in the case $p<q$ furthermore $x_{1}=0$, contradicting $Z \subset R$. In the case $p \leqq q, c_{2} \geqq 2$ the same argument works if $Z \subset \bigcup_{i=1}^{m} F_{p_{i}}$ but not contained in a single fibre.

The next two Propositions show, that every $\omega$-semi-stable and on non-algebraic $X$ every simple 2-bundle with $c_{1}=0,1 \leqq c_{2} \leqq 1+2 p_{g}$ occurs as an extension (*).

Proposition 1.7. Let $E$ be an $\omega$-semi-stable rank-2 bundle over $X$ with $c_{1}=0$, $1 \leqq c_{2} \leqq 1+2 p_{g}$. Then either there is an extension

$$
0 \rightarrow \mathcal{O} \rightarrow E \rightarrow J_{Z} \rightarrow 0
$$

with zero-dimensional $Z$ of length $c_{2}$, or there exists a line bundle $\mathcal{O}(D)$, $D \sim a F+\sum_{i=1}^{m} x_{i} F_{p_{i}}$ with $0 \leqq a, 0 \leqq x_{i}<p_{i}$ (i.e. $D$ is written in "normalized" form) and an extension

$$
0 \rightarrow \mathcal{O}(D-K) \rightarrow E \rightarrow J_{Z} \otimes \mathcal{O}(K-D) \rightarrow 0
$$

where again $Z$ is zero-dimensional of length $c_{2}$. In this case one has $0 \leqq a \leqq p_{g}+m-2$, so there is an $\mathcal{O}(D)$ maximal with respect to the $\omega$-degree; if the $p_{i}$ are pairwise relatively prime, the maximal $\mathcal{O}(D)$ is unique and satisfies $h^{0}(\mathcal{O}(2 D-2 K))=0$. For $c_{2}=1, Z=\{z\}$ is a simple point in the fixed locus of the linear system $|3 K-2 D|$; if furthermore the $p_{i}$ are pairwise relatively prime, the maximal $\mathcal{O}(D)$ and the accompanying point $z$ are uniquely determined by $E$ and satisfy $h^{0}\left(J_{z} \otimes \mathcal{O}(2 K-2 D)\right)=0, a \geqq p_{g}-1$.

Proof. The Riemann-Roch formula gives

$$
\chi(E)=-c_{2}+2+2 p_{g} \geqq 1,
$$

so either $E$ or $E(K)$ has a nontrivial section. In the first case, a 1-dimensional part in the zero set of the section would violate the semi-stability of $E$, hence we get the first extension. In the second case, take a section $s \neq 0$ in $E(K)$ to get an exact sequence

$$
0 \rightarrow \mathcal{O}(D-K) \rightarrow E \rightarrow J_{Z} \otimes \mathcal{O}(K-D) \rightarrow 0
$$

where $D$ is the 1-dimensional part of the zero-set of $s$, so $Z$ is zero-dimensional of length $c_{2}$.

Since $E$ is semi-stable we have

$$
0 \leqq \operatorname{deg}_{\omega}(\mathcal{O}(D-K))=\operatorname{deg}_{\omega_{0}}(D)+n D \cdot F-\operatorname{deg}_{\omega_{0}}(K)
$$


and because $D$ is a curve we get $D \cdot F=0$ by our choice of $n$. Writing $D \sim a F+\sum_{i=1}^{m} x_{i} F_{p_{i}}$ (normalized), $\operatorname{deg}_{\omega}(\mathcal{O}(D)) \leqq \operatorname{deg}_{\omega}(\mathcal{O}(K))$ reads

$$
a+\sum_{i=1}^{m} \frac{x_{i}}{p_{i}} \leqq p_{g}-1+\sum_{i=1}^{m} \frac{p_{i}-1}{p_{i}}
$$

or

$$
a+\sum_{i=1}^{m} \frac{x_{i}+1}{p_{i}} \leqq p_{g}-1+m
$$

giving the estimate for $a$. In particular, the degrees of possible $\mathcal{O}(D)$ are bounded, so there is a maximal one. If the $p_{i}$ are pairwise relatively prime, $\mathcal{O}(D)$ is determined by its degree and the maximal one is unique. Now assume $h^{0}(\mathcal{O}(2 D-2 K)) \neq 0$. Then we have

$$
\operatorname{deg}(\mathcal{O}(D-K))=\frac{1}{2} \operatorname{deg}(\mathcal{O}(2 D-2 K)) \geqq 0
$$

which together with $\operatorname{deg}(\mathcal{O}(D-K)) \leqq 0$ gives equality in $(* *)$, implying $\mathcal{O}(D)=\mathcal{O}(K)$ and thus leading back to the case $h^{0}(E) \neq 0$. At last, assume $c_{2}=1$ and $h^{0}\left(J_{z} \otimes \mathcal{O}(2 K-2 D)\right) \neq 0$. Mainly from the fact that $2 K-2 D$ is "even" one obtains $r \in\left\{p_{i}\right\}$ with $h^{0}\left(J_{z} \otimes \mathcal{O}\left(2 K-2 D-F_{r}\right)\right) \neq 0$. Now we tensor $(*)$ with $\mathcal{O}\left(K-D-F_{r}\right)$ to get

$$
0 \rightarrow \mathcal{O}\left(-F_{r}\right) \rightarrow E\left(K-D-F_{r}\right) \rightarrow J_{z} \otimes \mathcal{O}\left(2 K-2 D-F_{r}\right) \rightarrow 0
$$

and from $h^{0}\left(-F_{r}\right)=h^{1}\left(-F_{r}\right)=0$ we get $h^{0}\left(E\left(K-D-F_{r}\right)\right) \neq 0$, which violates the maximality of $\mathcal{O}(D)$. Since the line bundle associated to

$$
2 K-2 D \sim\left(2 p_{g}-2-2 a\right) F+\sum_{i=1}^{m}\left(2 p_{i}-2-2 x_{i}\right) F_{p_{i}}
$$

would have a section vanishing in $z$ if we had $2 p_{g}-2-2 a>0$, we get the lower bound for $a$.

For simple bundles we now consider a non-algebraic surface $X$, i.e. every curve $C$ in $X$ is vertical: If $C$ were not vertical, i.e. $C \cdot F>0$, then $(C+k F)^{2}>0$ for $k \gg 0$, hence $X$ is algebraic [2].

Proposition 1. 8. Let $E$ be a simple rank-2 bundle over a non-algebraic $X$ with $c_{1}=0,1 \leqq c_{2} \leqq 1+2 p_{g}$. Then the same conclusion holds as in the second case of Proposition 1.7. 
Proof. The simplicity of $E$ implies $h^{0}(E)=0$, so again Riemann-Roch gives $h^{0}(E(K)) \neq 0$, leading to the same extension as above; $D$ has to be vertical because $X$ is non-algebraic. The same argument as in the proof of Proposition 1.2 shows $h^{0}(\mathcal{O}(2 D-2 K))=0$. Writing $D \sim a F+\sum_{i=1}^{m} x_{i} F_{p_{i}}$ (normalized), we have

$$
2 D-2 K \sim 2\left(\left(a-p_{g}-m+1\right) F+\sum_{i=1}^{m}\left(x_{i}+1\right) F_{p_{i}}\right),
$$

so $h^{0}(\mathcal{O}(2 D-2 K))=0$ implies $a \leqq p_{g}+m-2$. The rest of the proof is as for Proposition 1. 7 .

Remark. For $m=1,2$ on non-algebraic $X$ there is no simple 2-bundle with $c_{1}=0, c_{2}=0$, because it would have a maximal divisor satisfying

$$
h^{0}(2 D-2 K)=h^{0}(2 K-2 D)=0 \quad \text { and } \quad h^{0}(3 K-2 D)>p_{g}+1
$$

which is impossible. This had to be expected for the following reason: If $E$ is an irreducible rank-2 Hermitian-Einstein bundle (see the introduction; such a bundle is $\omega$ stable and therefore simple) on $X$ with $c_{1}=0, c_{2}=0$, it is induced by an irreducible unitary representation of $\pi_{1}(X)$ of rank 2 [10], but for $m=2$ our $X$ has abelian fundamental group (see Section 2), so every irreducible unitary representation has rank 1.

\section{Moduli of bundles over simply connected elliptic surfaces}

Let $X$ be a minimal elliptic surface over $\mathbb{P}_{1}$ with multiple fibres $F_{p_{1}}, \ldots, F_{p_{m}}$ of multiplicities $p_{1}, \ldots, p_{m}$. The fundamental group of $X$ is abelian if and only if $m$ is at most 2 ; in this case the order of this group is g.c.d. $\left(p_{1}, p_{2}\right)$ [3]. Since multiple fibres can be removed using logarithmic transformations, we have the following characterization of simply connected minimal elliptic surfaces.

Proposition 2. 1. The simply connected minimal elliptic surfaces over $\mathbb{P}_{1}$ are precisely those surfaces which can be obtained by at most two logarithmic transformations with relatively prime multiplicities $p, q \geqq 1$ from a simply connected minimal elliptic surface without multiple fibres.

In the sequel $X_{p, q}$ will always denote an elliptic surface obtained by at most two logarithmic transformations of relatively prime multiplicities $p, q$ from an elliptic surface $X$ over $\mathbb{P}_{1}$ without multiple fibres. This surface $X$ is either rational $\left(p_{g}(X)=0\right)$, i.e. a Halphen pencil, or an elliptic $K 3$ surface $\left(p_{g}(X)=1\right)$ or properly elliptic. This follows immediately from the canonical bundle formula and the classification of compact surfaces [2].

$X_{p, q}$ is not necessarily algebraic, even if $X$ was algebraic. In fact, $X_{p, q}$ is algebraic if and only if it contains horizontal curves. On the other hand, by the work of Miyaoka [17], an elliptic surface has a Kähler structure if and only if its first Betti number is even. So we can always find a Kähler class on $X_{p, q}$. 
Proposition 2. 2. For all $p_{g} \geqq 0$ there exist surfaces $X_{p, q}$ without reducible fibres.

Proof. For any $d \geqq 1$ and any section $s$ of $\mathcal{O}_{\mathbb{P}_{1}}(d) \times \mathcal{O}_{\mathbb{P}_{2}}(3)$ over $\mathbb{P}_{1} \times \mathbb{P}_{2}$ take $X=(s)_{0}$ and let $\pi: X \rightarrow \mathbb{P}_{1}$ denote the induced projection. For generic $s, X$ will be smooth with irreducible fibres. From $K_{X}=\pi^{*}\left(\mathcal{O}_{\mathbb{P}_{1}}(d-2)\right)$ we see $p_{g}(X)=d-1$.

Remark. Using Freedman's results, it is easy to determine the topology of the surfaces $X_{p, q}$ [8], [15]. The homeomorphism type of $X_{p, q}$ is determined by the geometric genus $p_{g}$ alone if $p_{g}$ is even; it is determined by $p_{g}$ and the parity of $p+q$ if $p_{g}$ is odd. More precisely, let $X_{0}$ denote a Kummer surface and $\bar{P}_{2}$ the projective plane with orientation reserved. Then $X_{p, q}$ is homeomorphic to the connected sum

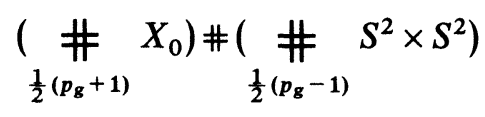

if $p_{g}$ is odd and $p+q$ even; in all other cases is $X_{p, q}$ homeomorphic to

$$
\left(\underset{2 p_{\mathbf{g}}+1}{\#} \mathbb{P}_{2}\right) \#\left(\underset{10 p_{\mathbf{g}}+9}{\#} \overline{\mathbb{P}}_{2}\right) \text {. }
$$

The surfaces $X_{p, q}$ with $p_{g}=1$ and $p+q$ even are the so called homotopy- $K 3$-surfaces, first studied by Kodaira [12].

For the rest of this section we denote by $X=X_{p, q}$ an elliptic surface over $\mathbb{P}_{1}$ with $p_{g} \geqq 1$, without reducible fibres and with at most two multiple fibres $F_{p}, F_{q}$ of relatively prime multiplicities $p, q \geqq 1$ (see Proposition 2. 2), so we can use the results of Section 1 . In particular, we fix a Kähler form $\omega$ on $X$ as in the remark before Corollary 1.4.

The first step to classify all semi-stable (simple) rank-2 bundles with $c_{1}=0, c_{2}=1$ on an algebraic (non-algebraic) $X$ is according to Propositions 1.7, 1.8 the following:

To find possible maximal vertical divisors defining semi-stable or simple bundles, determine all pairs $(D, z)$ where $D \sim a F+b F_{p}+c F_{q}$ (normalized) is a vertical curve with $p_{g}-1 \leqq a \leqq p_{g}, h^{0}(\mathcal{O}(2 D-2 K))=0$, and $z$ is a simple point in the fixed locus of the linear system $|3 K-2 D|$ such that $h^{0}\left(J_{z} \otimes \mathcal{O}(2 K-2 D)\right)=0$.

In principle this can be done for all pairs $(p, q)$, but we restrict ourselves to the cases $p=1,2,3$.

Proposition 2. 3. For $p=1,2,3$ the pairs $(D, z)$ with the properties just described are as follows:

i) For $p=1$ no such curve exists.

ii) For $p=2$ there are two different types:

$$
\begin{aligned}
& \text { I) } D \sim\left(p_{g}-1\right) F+F_{2}+c F_{q}, \quad z \in F_{2}, \frac{q-1}{2} \leqq c \leqq q-2, \\
& \text { II) } D \sim p_{g} F+c F_{q}, \quad z \in F_{2}, 0 \leqq c \leqq \frac{q-3}{2} .
\end{aligned}
$$


iii) For $p=3$ there are five types:

$$
\begin{aligned}
& \text { I) } D \sim\left(p_{g}-1\right) F+F_{3}+(q-1) F_{q}, z \in F_{q}, \\
& \text { II) } D \sim\left(p_{g}-1\right) F+2 F_{3}+c F_{q}, z \in F_{3}, \frac{q-1}{2} \leqq c \leqq q-2, \\
& \text { III) } D \sim p_{g} F+c F_{q}, \quad z \in F_{q}, \frac{q-2}{2} \leqq c \leqq q-2, \\
& \text { IV) } D \sim p_{g} F+F_{3}+c F_{q}, \quad z \in F_{q}, c \leqq \frac{q-4}{2}, \\
& \text { V) } D \sim p_{g} F+F_{3}+c F_{q}, \quad z \in F_{3}, c \leqq \frac{q-3}{2} .
\end{aligned}
$$

Proof. Tedious computation.

Proposition 2. 4. The classes of maximal divisors are:

for $p=2$.

i) $\quad \tilde{\mathrm{I}}) \quad D \sim\left(p_{g}-1\right) F+F_{2}+c F_{q}, z \in F_{2}, \frac{3 q-3}{4} \leqq c \leqq q-2$,

II) $D \sim p_{g} F+c F_{q}, \quad z \in F_{2}, \frac{q-3}{4} \leqq c \leqq \frac{q-3}{2}$

ii) II) $D \sim\left(p_{g}-1\right) F+2 F_{3}+c F_{q}, z \in F_{3}, \frac{5 q-5}{6} \leqq c \leqq q-2$,

IIII) $D \sim p_{g} F+c F_{q}, \quad z \in F_{q}, \frac{2 q-4}{3} \leqq c \leqq q-2$,

IV) $D \sim p_{g} F+F_{3}+c F_{q}, \quad z \in F_{q}, \frac{q-4}{3} \leqq c \leqq \frac{q-4}{2}$,

V) $\quad D \sim p_{g} F+F_{3}+c F_{q}, \quad z \in F_{3}, \frac{q-5}{6} \leqq c \leqq \frac{q-3}{2}$

for $p=3$.

Proof. i) Let $D, D^{\prime}$ be as in Proposition 2.3 ii) with $\operatorname{deg}(D)<\operatorname{deg}\left(D^{\prime}\right)$ and $E$ a bundle given by

$$
0 \rightarrow \mathcal{O}(D-K) \rightarrow E \rightarrow J_{z} \otimes \mathcal{O}(K-D) \rightarrow 0
$$

Tensor (*) with $\mathcal{O}\left(K-D^{\prime}\right)$ to get

$$
0 \rightarrow \mathcal{O}\left(D-D^{\prime}\right) \rightarrow E\left(K-D^{\prime}\right) \rightarrow J_{z} \otimes \mathcal{O}\left(2 K-D-D^{\prime}\right) \rightarrow 0 .
$$

Of course $\operatorname{deg}(D)<\operatorname{deg}\left(D^{\prime}\right)$ implies $h^{0}\left(\mathcal{O}\left(D-D^{\prime}\right)\right)=0$. If $D$ and $D^{\prime}$ are of the same type, one sees

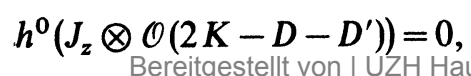


$E\left(K-D^{\prime}\right)$ has no section. If $D$ and $D^{\prime}$ are of different types, it is easy to check that $h^{0}\left(\mathcal{O}\left(K+D^{\prime}-D\right)\right)=p_{g}+1$, so $h^{1}\left(\mathcal{O}\left(D-D^{\prime}\right)\right)=0$ by Riemann-Roch and

$$
h^{0}\left(E\left(K-D^{\prime}\right)\right)=h^{0}\left(J_{z} \otimes \mathcal{O}\left(2 K-D-D^{\prime}\right)\right) .
$$

It follows that $D$ is maximal if and only if $h^{0}\left(J_{z} \otimes \mathcal{O}\left(2 K-D-D^{\prime}\right)\right)=0$ for all $D^{\prime}$ with $\operatorname{deg}(D)<\operatorname{deg}\left(D^{\prime}\right)$. Using this criterion the proof is by computation.

ii) Eliminate all $(D, z)$ in Proposition 2.3 iii) for which there exists a $D^{\prime}$ with $\operatorname{deg}(D)<\operatorname{deg}\left(D^{\prime}\right), h^{1}\left(\mathcal{O}\left(D-D^{\prime}\right)\right)=0$ and $h^{0}\left(J_{z} \otimes \mathcal{O}\left(2 K-D-D^{\prime}\right)\right) \neq 0$. To see that the remaining pairs $(D, z)$ are all maximal it suffices to check $h^{0}\left(J_{z} \otimes \mathcal{O}\left(2 K-D-D^{\prime}\right)\right)=0$ for every other remaining $D^{\prime}$ with $\operatorname{deg}(D)<\operatorname{deg}\left(D^{\prime}\right)$.

Corollary 2. 5. For $p=2,3$ and non-algebraic $X$ the isomorphism classes of simple rank-2 bundles on $X$ with $c_{1}=0, c_{2}=1$, are in one to one correspondence with pairs $(\mathcal{O}(D), z)$ where $(D, z)$ are as in Proposition 2. 4. The $\omega$-stable bundles over algebraic or non-algebraic $X$ are exactly those defined by $(D, z)$ satisfying $\operatorname{deg}(D-K)<0$. The $\omega$-semistable bundles which are not stable are exactly those defined by an extension

$$
0 \rightarrow \mathcal{O} \rightarrow E \rightarrow J_{z} \rightarrow 0
$$

where $z$ is a simple point in $F_{p} \amalg F_{q}$. .

Proof. Every simple bundle defines a unique maximal pair $(\mathcal{O}(D), z)$ by Proposition 1.8. Conversely, any $(D, z)$ in our list satisfies $h^{1}(\mathcal{O}(2 D-2 K))=0$, so by Proposition 1.1 it defines a unique bundle $E$. Since all divisors on the list satisfy the conditions of Proposition 1.2, E is simple.

From Proposition 1.3 and using the numerical criterion in Corollary 1.4 it is easy to check that the $\omega$-stable bundles are exactly those with $\operatorname{deg}(D-K)<0$, and that the remaining $D$ define bundles which are not even $\omega$-semi-stable. Thus the last statement follows from Proposition 1.7.

So far we have determined the moduli spaces as sets; now we are going to look at their algebraic (analytic) structures.

First let us consider the case where $X$ is algebraic. We take an ample line bundle $L_{X}$ on $X$, then for $n \gg 0$ the line bundle $L=L_{X} \otimes \mathcal{O}(n K)$ is again ample, and the Kähler form $\omega$ induced by $L$ has the properties we need to use the results of Section 1 . Denote by $M_{p, q}$ the moduli space of $L$-stable rank-2 bundles on $X_{p, q}$ with Chern classes $c_{1}=0$, $c_{2}=1$. For every pair $r_{1} \leqq r_{2}$ of rational numbers let $\left[r_{1}, r_{2}\right]$ denote the number of integers $m$ with $r_{1} \leqq m \leqq r_{2}$.

Theorem 2. 6. i) $M_{1, q}=\emptyset$.

ii) $M_{2, q}=\coprod_{\mu} F_{2}$ with $\mu=\left[\frac{3 q-3}{4}, q-2\right]+\left[\frac{q-3}{4}, \frac{q-3}{2}\right]$, in particular, $M_{2, q}$ is reduced.

iii) $\quad\left(M_{3, q}\right)_{\mathrm{red}}=\left(\amalg_{v} F_{3}\right) \amalg F_{q}$ with $v=\left[\frac{5 q-5}{6}, q-2\right]+\left[\frac{q-5}{6}, \frac{q-4}{3}\right]$. 
Proof. i) There exists no stable bundle on $X_{1, q}$ by Proposition 2. 3.

ii) For $p=2$ it is easy to see that every divisor in Proposition 2.4 i) defines a stable bundle, so there is an obvious one to one correspondence between pairs $(\mathcal{O}(D), z)$ and points in $\underset{\mu}{\amalg} F_{2}$. It remains to show that this is the moduli space in the schemetheoretical sense.

Consider an arbitrary component $F_{2}$ of $\coprod_{\mu} F_{2}$ and let $D$ be the corresponding divisor. If $\Gamma$ denotes the graph of the natural inclusion $F_{2} \subset X$, we have the following commutative diagram:

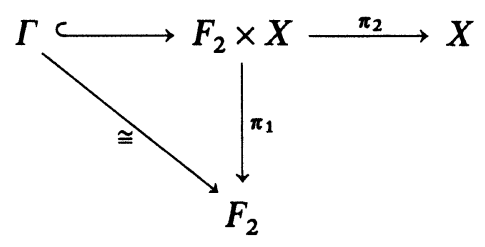

Let $L_{D}:=\operatorname{Ext}_{\pi_{1}}^{1}\left(J_{\Gamma} \otimes \pi_{2}^{*}(\mathcal{O}(K-D)), \pi_{2}^{*}(\mathcal{O}(D-K))\right)$, then from standard arguments [21] we find an extension

$$
0 \rightarrow \pi_{2}^{*}(\mathcal{O}(D-K)) \rightarrow E \rightarrow J_{\Gamma} \otimes \pi_{2}^{*}(\mathcal{O}(K-D)) \otimes \pi_{1}^{*}\left(L_{D}\right) \rightarrow 0
$$

on $F_{2} \times X$ where $E$ is locally free, and for every $z \in F_{2}$ this $E$ restricts on the $\pi_{1}$-fibre $\{z\} \times X$ to the bundle determined by $(D, z)$.

From the universal property of the moduli scheme $M_{2, q}$ we get a bijective morphism

$$
\coprod_{\mu} F_{2} \rightarrow\left(M_{2, q}\right)_{\mathrm{red}} .
$$

To see that $M_{2, q}$ is reduced it suffices to show $h^{1}($ End $E) \leqq 1$ for every $L$-stable bundle $E$. For this consider the sequence

$$
0 \rightarrow E(D) \rightarrow \text { End } E \otimes \mathcal{O}(K) \rightarrow J_{z} \otimes E(2 K-D) \rightarrow 0 .
$$

By the Riemann-Roch formula we have $\chi($ End $E)=4 p_{g}$, so we have to show $h^{0}($ End $E \otimes \mathcal{O}(K)) \leqq 4 p_{g}$. Since $h^{0}(E(D))=2 p_{g}$ for all $D$ in Proposition 2.4 i) we have to prove $h^{0}\left(J_{z} \otimes E(2 K-D)\right) \leqq 2 p_{g}$. But $h^{0}(E(2 K-D))=2 p_{g}+1$, so we need a section in $E(2 K-D)$ which does not vanish in $z$.

The restriction of $E(2 K-D)$ to $F_{2}$ splits:

$$
\left.E(2 K-D)\right|_{F_{2}}=\left(\mathcal{O}_{F_{2}}(z) \oplus \mathcal{O}_{F_{2}}(-z)\right) \otimes N_{F_{2} / X}^{*}
$$

where $N_{F_{2} / X}^{*}$ is the non-trivial conormal bundle of $F_{2}$ in $X$. Thus there is a unique section of $\left.E(2 K-D)\right|_{F_{2}}$ not vanishing in $z$. This section lifts to $E(2 K-D)$ if and only if $h^{0}\left(E\left(2 K-D-F_{2}\right)\right) \leqq 2 p_{g}$ which is equivalent to $h^{0}\left(J_{z}\left(3 K-2 D-F_{2}\right)\right) \leqq p_{g}$. This can be verified by calculation. 
iii) The restrictions on the values $c$ in Proposition 2.4 ii) coming from $\operatorname{deg}(D)<\operatorname{deg}(K)$ are for the different types as follows:

$$
\begin{aligned}
& \text { II) } \frac{5 q-5}{6} \leqq c \leqq q-2 \\
& \text { III) } c=\frac{2 q-4}{3}(\text { so } q=2(\bmod 3)) \\
& \text { IV) } c=\frac{q-4}{3}(\text { so } q=1(\bmod 3)) \\
& \text { V) } \frac{q-5}{6} \leqq c \leqq \frac{q-4}{3}
\end{aligned}
$$

This gives $M_{3, q}$ as a set; the construction of the universal family $E$ is as above. But using the same kind of arguments as for $p=2$ one sees that almost all components of $M_{3, q}$ are non-reduced.

Now let $X$ be non-algebraic and denote by ${ }^{s} M_{p, q}$ the analytic moduli space of simple rank-2 bundles on $X$ with $c_{1}=0, c_{2}=1$.

Theorem 2. 7. i) ${ }^{s} M_{1, q}=\emptyset$.

ii) ${ }^{s} M_{2, q}=\coprod_{\mu} F_{2}$ with $\mu=\left[\frac{3 q-3}{4}, q-2\right]+\left[\frac{q-3}{4}, \frac{q-3}{2}\right]$.

iii) $\quad\left({ }^{s} M_{3, q}\right)_{\mathrm{red}}=\left(\coprod_{\lambda_{1}} F_{3}\right) \amalg\left(\coprod_{\lambda_{2}} F_{q}\right)$ with

$$
\begin{aligned}
& \lambda_{1}=\left[\frac{5 q-5}{6}, q-2\right]+\left[\frac{q-5}{6}, \frac{q-3}{2}\right], \\
& \lambda_{2}=\left[\frac{2 q-4}{3}, q-2\right]+\left[\frac{q-4}{3}, \frac{q-4}{2}\right] .
\end{aligned}
$$

Proof. Set-theoretically this is again obtained from Proposition 2. 4 and Corollary 2. 5; the construction of the universal family and the proof of the reducedness is as above.

\section{We close with a final}

Remark. We have ${ }^{\omega} M_{p, q}={ }^{s} M_{p, q}$ for $p=1,2$, whereas there are many components in ${ }^{s} M_{p, q} \backslash{ }^{\omega} M_{p, q}$ for $p=3$. The description of ${ }^{\omega} M_{p, q}$ is th same as of $M_{p, q}$ for algebraic $X$, even if $\omega$ is not induced by an ample line bundle. Moreover, the moduli spaces are independent of the Kähler form $\omega_{0}$ (resp. the ample line bundle $L_{X}$ ) we started with to get $\omega$ (resp. $L$ ). In the algebraic case there may exist simple bundles which are not defined by vertical divisors. 


\section{References}

[1] C. Bănică, M. Putinar, G. Schumacher, Variation der globalen Ext in Deformationen kompakter komplexer Räume, Math. Ann. 250 (1980), 135-155.

[2] W. Barth, C. Peters, A. van de Ven, Compact complex surfaces, Erg. Math. 4, Berlin-HeidelbergNew York 1984.

[3] I. Dolgachev, Algebraic surfaces with $q=p_{g}=0$, C.I.M.E. (1981).

[4] S. K. Donaldson, Anti self-dual Yang-Mills connections over complex algebraic surfaces and stable vector bundles, Proc. London Math. Soc. 50 (1985), 1-26.

[5] S. K. Donaldson, The differential topology of complex surfaces, C.R.A.S. 301, (1) $n^{\circ} 6$ (1985), 317.

[6] S. K. Donaldson, Connections, cohomology and the intersection forms of 4-manifolds, J. Diff. Geom. 24 (1986), 275-341.

[7] D. S. Freed, K. K. Uhlenbeck, Instantons and four-manifolds, M.S.R.I. Publ. 1, Berlin-HeidelbergNew York 1984.

[8] M. Freedman, The topology of four dimensional manifolds, J. Diff. Geom. 17 (1982), 357-453.

[9] S. Kobayashi, Curvature and stability of vector bundles, Proc. Japan Acad. 58 A4 (1982), 158-162.

[10] S. Kobayashi, Differential geometry of holomorphic vector bundles, PAM 315, Berkeley 1986.

[11] K. Kodaira, On the structure of compact complex analytic surfaces. I, Am. J. Math. 86 (1964), 751-798.

[12] K. Kodaira, On homotopy-K 3 surfaces. Essays on topology and related topics (ded. G. de Rham), BerlinHeidelberg-New York 1970.

[13] M. Lübke, Stability of Einstein-Hermitian vector bundles, manuscripta math. 42 (1983), 245-257.

[14] M. Lübke, C. Okonek, Moduli spaces of simple bundles and Hermitian-Einstein connections, Math. Ann. 276 (1987), 663-674.

[15] R. Mandelbaum, On the topology of elliptic surfaces, Adv. math. suppl. studies 3 (1979).

[16] M. Maruyama, Moduli of stable sheaves. II, J. Math. Kyoto Univ. 18 (1978), 557-614.

[17] Y. Miyaoka, Kähler metrics on elliptic surfaces, Proc. Japan Acad. 50 (1974), 533-536.

[18] S. Mukai, Symplectic structure of the moduli space of sheaves on an Abelian or $K 3$ surface, Invent. math. 77 (1984), 101-116.

[19] A. Norton, Analytic moduli of complex vector bundles, Indiana Univ. Math. J. 28 (1979), 365-387.

[20] C. Okonek, M. Schneider, H. Spindler, Vector bundles on complex projective spaces, Progress math. 3, Boston 1980.

[21] C. Okonek, A. van de Ven, Stable bundles and differentiable structures on certain elliptic surfaces, Invent. math. 86 (1986), 357-370.

[22] C. H. Taubes, Self-dual connections on 4-manifolds with indefinite intersection matrix, J. Diff. Geom. 19 (1984), 517-560.

[23] K. Uhlenbeck, S. T. Yau, On the existence of Hermitian-Yang-Mills connections in stable vector bundles, preprint 1986.

Mathematisch Instituut, Rijksuniversiteit Leiden, Niels Bohrweg 1, NL-2300 RA Leiden

SFB 170, Mathematisches Institut, Bunsenstraße 3-5, D-3400 Göttingen

Eingegangen 16. Juni 1986 